\title{
Conceptual or procedural mathematics for engineering students - views of two qualified engineers from two countries
}

\author{
Christer Bergsten $^{\mathrm{a}}$, Johann Engelbrecht ${ }^{\mathrm{b} *}$ and Owe Kågesten ${ }^{\mathrm{c}}$ \\ ${ }^{a}$ Department of Mathematics, Linköping University, Linköping, Sweden; ${ }^{b}$ Department of Science, \\ Mathematics and Technology Education, University of Pretoria, Pretoria, South Africa; \\ ${ }^{c}$ Department of Science and Technology, Linköping University, Linköping, Sweden
}

This study forms part of a collaboration project between universities in South Africa and Sweden in which we investigate whether the emphasis in undergraduate mathematics courses for engineering students would benefit from being more conceptually oriented than a traditional more procedurally oriented way of teaching. In this paper, we report in some detail from two interviews with professional engineers, selected to represent two different 'poles' of engineering work. The aim was to explore different kinds of arguments regarding the role of mathematics in engineering work, as well as some common across contexts. Both interviewees feel that conceptual mathematics is more important for engineering work, although the role of the procedural aspect was seen by one of the interviewees also to be important, but in a very intricate way.

Keywords: engineering mathematics; practising engineers; conceptual and procedural knowledge; interviews

\section{Introduction}

According to Kent and Noss, $[1, \mathrm{p} .8]$ mathematics 'is and will remain crucial' for engineering education. It is not clear, however, how the mathematical education of engineers should be organized. For example, the types of mathematical knowledge engineers need, changes of practice and education suggested by the advance of computer technology, or how and when mathematics should be taught, are still discussed in the literature.[1,p.8] Considering the increased use of computing technology, one issue with specific relevance for engineers concerns the balance between procedural fluency (or calculation skills) and conceptual insight in connection to mathematical work, not only in professional contexts but also during the education of engineers. This paper explores this issue by analysing interviews with two practising engineers where the focus was on their views on the relevance of procedural and conceptual aspects of mathematics in their work and in the education of engineers.

\section{Background}

In his review of studies on the use of mathematics in engineering workplaces, Alpers identified several categories that "capture the way mathematical thinking or activities occur during

*Corresponding author. Email: jengelbr@up.ac.za 
the work on practical problems'.[2,p.3] One category concerns the contextual embedding of mathematical models, concepts and procedures; another involves a recognition that the operation of technological tools still requires understanding of mathematical notations and graphics; while another category points at an appreciation that mathematics is important and necessary but not sufficient for engineering design, where also 'engineering judgement' and 'sceptical reverence' are critical (cf. [3]). A similar wide approach to the mathematical needs of engineers is found in [4]. In line with these views, the employers interviewed in the study by Kent and Noss express more emphasis on what they call an 'holistic' awareness of the mathematical needs for engineering work than on 'manipulative skill'.[1,p.9]

Kent and Noss [1] conducted interviews with practicing engineers at industries and mathematicians teaching engineering mathematics. While the findings pointed to an extensive use of mathematical concepts, models and computing tools, the engineers did not do much routine calculation but needed to communicate mathematical ideas. This last issue has been addressed by other authors as well (e.g. [5]). In an interview study of qualified mathematical science graduates, Wood [6] found that these graduates had to learn other mathematics than was taught in their education when employed, as well as more computing skills to meet workplace demands. According to Henderson and Broadbridge,[7] in an Australian context, most professional and academic engineers agree that engineers need a 'good grounding in mathematics', logic and problem solving. The authors raise a concern about a lack of confidence in using mathematics among many professional engineers.

In her survey on the views of academics, industry personnel and students in Australia on the essential generic and specialist skills and attributes of a modern engineer, Nguyen found that technical knowledge and skills, including knowledge about 'how to behave and operate within an organisation',[8,p.73] as well as attitudes, are essential. Together with the brief survey of the literature above, this points to the need to view the mathematical education of engineers in a wider perspective, including conceptually and procedurally oriented teaching and learning of mathematics, than with a focus only on mathematical content issues.

\section{Conceptual and procedural approaches in mathematical work}

At many universities, mathematics studies in engineering education begin with calculus and linear algebra courses, often taught at mathematics departments that treat their subject as valuable knowledge in its own right, rather than as a service subject for practical use outside mathematics. In this context, there is a strong tradition regarding the content of these courses as well as teaching approaches used [9]. However, even within this 'traditional' approach to mathematics teaching, there is a difference between taking a mainly conceptual or procedural approach. Teaching with a focus on conceptual understanding may start by providing a contextual basis for the new mathematical knowledge requiring students to connect to their prior knowledge.[10] Taking a more procedural approach, the lecturer may present definitions, notations and procedures without first providing meaningful contexts to concepts and methods involved.

While the distinction between these two aspects of doing, teaching and learning mathematics has prevailed, it has also been criticized. In mathematics education, this distinction between conceptual and procedural knowledge was much discussed in the 1980s, mainly within a psychological framework with reference to Piaget and mental schemes. Hiebert and Lefevre defined conceptual knowledge in mathematics as a connected network of knowledge in which 'the linking relationships are as prominent as the discrete pieces of information'.[11,p.3-4] As an example of conceptual knowledge, they note the construction 
of a relationship between the algorithm for multi-digit subtraction and knowledge of the positional place values of digits.[11]

Procedural knowledge was defined by two parts, constituted by step-by-step procedures for solving mathematical tasks, on one hand, and knowledge of the symbolic representations used in such procedures, on the other hand. To be competent in mathematics, then involves not only of knowledge of concepts and knowledge of procedures but also of relations between these two types of knowledge.[12]

Research suggests that there is a complex interplay between conceptual and procedural knowledge [13,14]; conceptual knowledge may influence or even become procedural with repeated exposure, while procedural knowledge may support the development of conceptual knowledge.[15,16] Some authors see these two constructs as independent,[17-19] while others have emphasized dynamic and evolutionary aspects between them (e.g. [20-23]). Gray and Tall [24] coined the term 'procept' for an integrated knowledge of processes and concepts that is characteristic for successful learners of mathematics. Kieran [25] claims, however, that the distinction is a false dichotomy, with reference to the nature of algebraic symbols. Also, in the context of more advanced mathematics, Wu argues from a number of examples that 'in mathematics, skills and understanding are completely intertwined'.[26,p.2]

In the context of mathematics in engineering education, the relation between conceptual and procedural approaches to solving mathematics tasks has been investigated within the larger project for the present study. That this relation is highly complex was illustrated by Engelbrecht, Bergsten and Kågesten, who found that first-year engineering students tended to 'proceduralize' tasks designed to have a conceptual focus, sometimes requiring "quite sophisticated mathematical work".[27,p.939] The study by Engelbrecht, Bergsten and Kågesten [28] on junior engineering students' achievement and views on conceptually versus procedurally focused mathematics tasks showed that the students considered procedural questions as more common in their mathematics curriculum, while conceptual (mathematical) questions were seen as more common in engineering subjects; they also expressed a higher confidence in their performance on the procedural tasks. However, both types of tasks were seen as relevant for their engineering studies. In [29], strong correlations between performance and confidence for the procedural items for junior and senior students were found, while for the conceptual items, these correlations were much stronger for the senior than for the junior students. This points to an increased familiarity with the conceptual aspects of mathematics through its use in applied subjects throughout the education.

\section{Methodology}

This study is part of a collaboration project between two universities, one in South Africa and one in Sweden. We address various populations' - engineering students', lecturers' and practising engineers' - views on the role of mathematics in engineering education with a particular focus on conceptual/procedural approaches. In earlier parts of the project, we have problematized the distinction between conceptual and procedural approaches,[27] and surveyed junior (second year) and senior (fourth year) engineering students from both countries.[28,29]

In the overall project as well in this study, we use the following working definitions regarding the approach to the mathematical education of engineers (cf. [30]): A conceptual approach includes translations between verbal, visual (graphical), numerical and formal/algebraic mathematical expressions (representations); linking relationships; and 
interpretations and applications of concepts (for example, by way of diagrams) to mathematical situations. A procedural approach includes (symbolic and numerical) calculations, employing (given) rules, algorithms, formulae and symbols.

The aim of this study was to explore views and arguments concerning the role of conceptual versus procedural aspects of mathematics in engineering work and education put forward by professional engineers. Twenty-five qualified engineers who are practising as actual engineers, engineers in managerial positions and university lecturers in applied (engineering) subjects were interviewed.[31] We selected experienced engineers who had initial training as engineers at academic universities and were from a range of engineering areas, using a combination of convenience and snowball sampling (see [32,p.100]).

The research question, How do professional engineers view the relevance and role of conceptual and procedural mathematical skills in the education and practices of engineers?, was used as a guide to structure the interviews and the analysis of the interview protocols.

In this paper, we report, as a case study, in some detail from two of these interviews [see [31] for the full results from different engineers], selected to represent two different 'poles' of engineering work, one being more on research and product development oriented and one more on site practice oriented. By looking at these different cases, which may represent both, what Bryman calls a unique case and an exemplifying case,[32,p.51] we hoped to be able to find different kinds of arguments regarding the role of mathematics in engineering work, as well as some that were common across contexts.

The interviews were audio-recorded and transcribed. The interview with the South African engineer was conducted in English, while the interview with the Swedish engineer was conducted in Swedish; the interview protocol was then translated into English. The interview transcripts were analysed by the researchers in collaboration.

In the semi-structured interviews,[32,p.321] the concepts of conceptual and procedural approaches were initially explained to the interviewees, using our working definitions and a few examples of items from the research instrument used with the students. To investigate the research question above, the interviews were organized by the following guiding questions:

From the point of view of your current professional activities, what is your opinion on your own mathematical schooling from your engineering education?

Which approach is more relevant for the work you do (procedural or conceptual)?

If you were involved in training engineers, which approach would you use?

Should engineers that you would employ have conceptual or procedural skills?

For the analysis of the two interview protocols, a matrix was set up where the answers from each interviewee were organized in columns sorted by questions. By identifying content categories of responses in a column across the two interviewees, similarities and differences between their views were possible to describe for each question. A picture of the overall view of each interviewee on the procedural/conceptual issue was obtained by a two-step coding procedure, with specific codes sorted within overarching themes. We thus tried to combine basic elements from grounded theory with a narrative thematic approach to identify the overall position of the interviewee on the different issues discussed [32,p.401-404, 412]; the coding scheme is presented in Figure 1. Although the interview was of a 'standard' semi-structured type, a narrative analysis could thus be performed of the interview transcript using a thematic approach.[32,p.412] The presentation of the interview 


\begin{tabular}{|c|c|c|}
\hline Engineer & Themes & Specific codes \\
\hline \multirow{3}{*}{ Robert } & Language & $\begin{array}{l}\text { mathematical language, learn the language, a } \\
\text { little mechanical, the foundation, this calculating }\end{array}$ \\
\hline & Understanding & $\begin{array}{l}\text { certain value to it, structure the problem, } \\
\text { independent, self-sustaining, the formulation } \\
\text { and problem-solving way }\end{array}$ \\
\hline & Both important & $\begin{array}{l}\text { a little so little so, a little mix in between } \\
\text { ['language' and 'understanding'], both are } \\
\text { important, don't believe in any of the extremes }\end{array}$ \\
\hline \multirow{3}{*}{ Ben } & Procedural & $\begin{array}{l}\text { very procedural, calculate this, procedural } \\
\text { guys, mathematics is procedural, we don't need } \\
\text { the procedural mathematics, they have done } \\
\text { their steps then this is the answer }\end{array}$ \\
\hline & Conceptual & $\begin{array}{l}\text { the conceptual one, fit the line through that, we } \\
\text { see this is a nice curve that fits, understands } \\
\text { this graph, conceptual guys, the whole } \\
\text { conceptual understanding, did not understand, } \\
\text { someone who understands what the answer is, } \\
\text { that is conceptual, a feel for the answer, you } \\
\text { need to know if it is realistic }\end{array}$ \\
\hline & Not much used & $\begin{array}{l}\text { [mathematics is] very little used, it falls away, } \\
\text { we use very little of it }\end{array}$ \\
\hline
\end{tabular}

Figure 1. Themes and codes used in the analysis of the two interview protocols.

data will, therefore, be in a narrative format, followed by a discussion of the outcome of the thematic analysis.

\section{Interview 1 - the Swedish engineer Robert}

\subsection{Background}

After his engineering education, which led to a master's level exam in the area of technical physics and electric engineering, Robert continued to earn a doctorate in experimental physics with a follow-up postdoc year. Since then he has been working in different management, product and business development positions for more than two decades in a big company producing advanced industry material. He has also been involved in international collaborations. Product development he found 'very exciting, you start from a blank sheet of paper and try to shape something to a product then finally'.

\subsection{Summary of interview 1}

To the opening interview question, How do you see your mathematical education from the perspective you have today?, Robert describes mathematics as both a language and an art, emphasizing that he has not been much involved in computational activities.

Firstly, it's a language to be learned, mathematical language, and then it it's also a little art, I mean you must have some, you have to have imagination to solve these problems sometimes, and that one can learn mechanically but some have the aptitude for this, and some may work hard on it and so. And I have not solved any differential equation ago since I started here, I can say, I have not, I'm not doing computations but I have led persons doing computation. 
He refers to colleagues doing the computations for which 'they've been in their computing software'. Giving 'FEM' (finite element methods) as one mathematical method as an example of what for him seems to be how mathematics is used, he talks about 'the mathematics of it' as its procedural structure. Thus, in his 'professional life', Robert goes on:

I have not had use of, it is wrong to say that I don't have any use, it's good to have the insight but I feel that I have not used the mathematics of it

He describes, however, that his mathematical education has provided him with some sense of overview and control through a basic mathematical understanding.

I think the schooling itself, this with the need to learn this language, you have to, you have to be careful otherwise it will be wrong and that part, I think that's quite useful to go through that struggle, to be organized and accurate and be able to set it up [...] You get an understanding of, yes it is hard to say but I have not solved any differential equations since I started here. .. and no integrals either, no.

Robert thus expresses a value inherent in the mathematical training he has received during his education, even though he does not 'use it' in terms of performing calculations; it is rather the 'understanding' that he has developed that has been useful in the specific professional activities as an engineer required by the positions he has had over the years. Colleagues, he says, have done the mathematical calculations needed, using computer software.

When asked about the emphasis on procedural or conceptual aspects of mathematics during engineering education, Robert says the he believes

a little on, on both parts [...] you have to learn these, it will be a little mechanical perhaps, but I think it, it has a certain value to it too, it has a value, then I think that the other world is of course perhaps more closer to reality then.

While having some difficulties to pinpoint exactly why the procedural part of his mathematical experience is needed, Robert still hangs on to its importance even if he does not explicitly use it in his own work:

I think that you can't be simply a .... a thinker who can look at a graph and see that so and so this should be related. I also think that there is a certain mechanical, I don't know why but I feel that it is of some importance, I think. So I think you should have a little of, some mix [... I don't believe in any of the extremes.

He also sets up an order of things, starting with learning 'the mathematical language' (as in the quotes above), so that the (conceptual) understanding has something to grow out from, eventually to be able to make own judgements:

I think, especially in the beginning, I think you need to get this schooling and learn the language, then maybe later, you move more and more into this [...] the more understanding part there by yourself, be able to see the graphs and interpret and make your own judgments and so. Mm, maybe a bit like that.

That he adds 'maybe a bit like that' to this description indicates some uncertainty, as does the kind of excuse he puts forward by the words 'maybe it's because I'm just too traditional'. 
He is very explicit in expressing his view on the role of procedural mathematics and that it is needed as a basis for doing mathematics: 'I think you need to have the foundation. .... I think it is important.'

Regarding the relevance of procedural versus conceptual mathematics for his present job, Robert continues to talk in rather general terms, acknowledging that he does not deal much with mathematics directly.

\section{It's probably more about, creativity, and see the possibilities, the job I have today [...] maybe a little more towards, instead of this calculating so maybe a little more to the formulation and problem-solving way, I would say. But perhaps it is far from that as well.}

Despite the uncertainty expressed here, he again emphasizes that 'one must be accurate and can't be sloppy' and that 'both are important', but that the key issue is problem solving, 'in order to formulate the problems and be able to see something and structure and sort out'. It is clear that a conceptual approach, expressed through seeing structure and order, is more relevant for his own work than managing the calculations.

To the question about which kind of mathematical training, in terms of a procedural or conceptual emphasis, he would prefer his job applicants to have, he starts talking, however, about other types of competencies before coming to mathematics:

When you hire someone, as the first criterion, I think is the, first and foremost, has passed his training $[. .$.$] then I think it is important how to, how to work in a group as an individual, and$ then also skills of course

About the mathematical aspects Robert is very clear about his preferences, with reference to how it works today when you engage in a professional task as an engineer. It is not just about 'counting' on something.

And then I think, after all, this conceptual, conceptual, it doesn't work like that now that you put somebody into a corner and then "count on this", but it's more that "this is an area, solve this task", so of course, so it ends up more about it, solve this task and formulate the problem yourself and control this yourself, I think.

What he values is independence, and for that it is necessary to be able to deal with the whole problem situation. This is something that can be prepared during the education.

And I think that if you learn to work like that with math problems, you can surely apply it to other, other problems too. Being able to sort out the problem and in a way structured attack it and solve it, I think is important.

According to Robert, this is also what you do, to some extent, in, for example, a calculation where you insert a substitution to be able to finalize it. One reason for the need of independency is that there is normally no supervision of your work as support:

Those that require one constantly, telling you to go there, go there, go there, it doesn't work, well, not today, I think, but you want to have individuals who are independent and come up with their own ideas and solutions to problems, they are the best so to speak.

In education, Robert thus suggests to stimulate independence and self-confidence, and to be able to collaborate with others on challenging problems, not sitting with 'the thumb in the answer key and looking'. 


\section{Interview 2 - the South African engineer Ben}

\subsection{Background}

Ben qualified as a civil engineer at a major South African university with highway engineering as a specialist field, working specifically on road design, geometric design, pavements and structures. He has a long experience of engineering and is currently working with a firm of consulting engineers in a large engineering company in South Africa.

\subsection{Summary of interview 2}

To the opening question, From the point of view of your current professional activities, what is your opinion of your mathematics education that you obtained from your engineering degree?, Ben comments that it is 'very little used':

My applied maths was worth much more in the beginning and after 10 years in the industry it falls away, even in the good old days with very little computer ability. In road design for example, we lost it completely. We calculate coordinates, how to get from this point to that point, what is the distance between these two coordinates.

He says that the mathematics courses he took at the university were very procedural, while the work he does now is very conceptual.

The mathematics was: do this, prove this, and calculate this and that is not what we use here, well we use very little of it, especially with the software that you've got now.

In fact, he says, they do not use much of any of those types of mathematics; rather, the structural engineers 'do a lot more mathematics'.

To the question about which of the two approaches to mathematics (conceptual/ procedural) is most relevant to his current work, Ben answers shortly 'the conceptual one'. As an example, he describes how mathematics is involved in material testing:

A lot of these pavement or material tests that we do, you do a few tests and then you plot it in on a graph. You then draw a line and extrapolate it (hopefully not too far), but we fit the line through that and that's conceptual. We do not really care what the formula of this graph is, or what the numbers are. We plot it on one or two different scales and we see this is a nice curve that fits and there is the result.

He comments that this is all at the level of a technician with not very high qualifications in mathematics. It is more about understanding a graph, recognizing, for example, a parabolic curve, and being able to read off an answer without having to consider the actual calculations.

When asked about desired skills when hiring engineering graduates - very good procedural mathematical skills or very good conceptual mathematical skills - Ben finds it difficult to think in those terms in practice but that they would look for people who can think and solve the problems and find where the mathematics fit in.

I think probably conceptual, but it is not something that you can really measure. So we look for the bright boys and girls. But I will say conceptual is definitely that we need, because that is what the industry actually needs.

In discussing job opportunities for graduates with less conceptual skills but having mainly strong procedural abilities, Ben makes a distinction between what he calls 'brilliant guys' and 'bright guys': 
Engineering is an extremely wide field and in my opinion I don't want the brilliant guys, I want the bright guys. I think the conceptual guys are the bright guys and the procedural guys are probably the brilliant guys and there is room for them as well.

He adds, however, that there is scope also for other categories, because 'road design is more of an art, while structural design is engineering, it is a different level'. There a more heavy mathematics is used,

doing projections, transport economics and these sort of things. They count vehicles and they project vehicles in the future. It is also a different level so we can use all of them.

There is also a discussion about a possible lack of conceptual mathematics abilities in new engineering graduates coming into a company. Ben sees problems but not so much relating to mathematics per se:

I doubt whether the problems are at a mathematical level. I think it is a different level at some of the other subjects. We do mathematics for two years and that is not rocket science at that stage. So no, I don't blame the mathematics for the type of problems that we have. The whole conceptual understanding is lacking at an engineering level and not a mathematical level.

He contends that with a more conceptual exposure, they will understand a little bit more about work in the end, but that it is not the fact that mathematics is procedural at this stage that is the problem. However, if there was more conceptual work implemented, he would expect to see a difference. In the final comments, Ben is very explicit about the benefits of a more conceptual approach than the way mathematics is currently taught in engineering education, and that procedural mathematics is not really needed any more, due to the computing technology that is now available. He refers to

the applied mathematics that we did at university. We did not understand a word about it, it was very difficult. But in the end it is worth a lot more than the simple integration and differentiation.

Ben thus makes a connection between conceptual mathematics and applied mathematics. He also points out that regarding the importance of procedural mathematics, the development of computing technology has made a difference.

There was a big change when I finished studying; these small programmable calculators came out [...] and I think that is the main difference. We don't need the procedural mathematics. What we need now from these young guys is someone who understands what the answer is and to me that is conceptual.

This is, according to Ben, the problem with the young graduates today - that 'they have no idea'. Such type of understanding is partly based on experience and partly on what is being taught. When working with a graph, for example, and getting a number, it is necessary to be able to judge if it is realistic or not, something Ben does not think they are taught to do.

They think that if they have done their steps then this is the answer and whether they do it mathematically on a piece of paper or on a computer they do not have a feel for the answer. 


\section{Discussion}

The two engineers interviewed for this study have very different backgrounds and are working in different areas and types of engineering. Already these examples thus highlight the potential complexity in designing what could be called 'engineering education', and in particular its mathematical component. Nevertheless, a common theme clearly visible throughout both narratives was an emphasis on the need of a conceptual mathematical approach to the engineering work in which the interviewees were involved. In their practice, another common trait was that these engineers did not perform much mathematical activities in terms of actual calculations. These observations reflect the outcomes in [1]. Both Ben and Robert expressed a main concern about understanding what 'it is all about' rather than keeping track of computational details, something generally taken care of by software technology. The term 'conceptual' here seemed to be interpreted as judging graphical and numerical outcomes as realistic and viable in relation to the problem studied. While this interpretation aligns well with our working definition of having a 'conceptual approach', it also links to the notions engineering judgement and sceptical reverence.[3]

Another theme present throughout Robert's narrative was the rather implicit but important role of a procedural foundation underpinning the crucial conceptual mathematical understanding. Robert was struggling to articulate this complex but fundamental relationship between procedural and conceptual knowledge, something which is mirrored in the research literature as presented above. His expression 'one must be accurate and can't be sloppy' points to a competence he may have conceptualized as stemming from procedural skills developed during the mathematical training in engineering education. These views are in sharp contrast to Ben's very explicit description of the role of procedural approaches: 'We don't need the procedural mathematics'. He also makes a clear distinction between graduates with predominantly conceptual skills as 'bright guys' and graduates with procedural skills as 'brilliant guys'. This difference in views between Ben and Robert may well be linked to the different types of engineering work in which they are involved, as well as their very different backgrounds. Even if Ben points to other areas of engineering where the 'brilliant guys' are needed, the difference has implications for engineering curriculum conceptions. Ben said that his own mathematical education was very procedural and that it is very little used, while Robert, also saying he is using very little of the mathematics he studied, still values the procedural training he went through.

Taking into account Ben's view that procedural mathematics is no more needed, computational details being secured by computers, a decrease of procedural knowledge among engineers may well develop from an over-reliance on technology. To what extent this could become a problem is still an open issue.

Both Ben and Robert seem to give priority also to other generic engineering skills than the purely mathematical (cf. [8]), be it procedural or conceptual. Here Ben points to a more general engineering conceptual understanding, while Robert emphasizes independent work, self-confidence and judgement. The overall educational message from these engineers we interpret as a strong concern for an increased conceptual approach, not only to mathematics but to engineering education as a whole, to better balance the need from workplace engineering. This message also echoes well the voices from the other 23 engineers who were interviewed in the overall study described above.[31]

Still, while the selection of the interviewees aimed to cover a wide spectrum of types of engineering work, the diversity and development of this professional field can never be 'covered' in any reasonable sense by one sample. By providing a 'thick description' [33] of the arguments put forward in relaxed conversation settings by experienced engineers 
from different professional contexts, we hope to have pointed to some key concerns about the issue discussed relevant to their work. However, the intricacy of the relation between the procedural and conceptual aspects of mathematical work, as it has been described in these interviews, will need a continued empirical and epistemological attention in research to better understand the role of procedural knowledge in mathematics for engineering curriculum design.

\section{Disclosure statement}

No potential conflict of interest was reported by the author.

\section{References}

[1] Kent P, Noss R. Mathematics in the university education of engineers. London: Ove Arup Foundation; 2003. (Ove Arup Foundation Report). Available from: http://www.lkl.ac.uk/research/ REMIT/Kent-Noss-report-Engineering-Maths.pdf

[2] Alpers B. Studies on the mathematical expertise of mechanical engineers. J Math Model Appl. 2010;1(3):2-17.

[3] Gainsburg J. The mathematical disposition of structural engineers. J Res Math Educ. 2007;38:477-506.

[4] Cardella ME. Which mathematics should we teach engineering students? An empirically grounded case for a broad notion of mathematical thinking. Teach Math Appl. 2008;27(3):150159.

[5] Vest D, Long M, Anderson T. Electrical engineers' perceptions of communication training and their recommendations for curricular change: results of a national survey. IEEE Trans Prof Commun. 1996;39(1):38-42.

[6] Wood LN. Graduate voices: the nexus between learning and work [Unpublished PhD dissertation]. Sydney: Macquarie University; 2006.

[7] Henderson S, Broadbridge P. Engineering mathematics education in Australia. MSOR Connect. 2009;9(1):12-17. Available from: http://mathstore.ac.uk/headocs/9112_henderson_s_and_ broadbridge_p_engmathed.pdf.

[8] Nguyen DQ. The essential skills and attributes of an engineer: a comparative study of academics, industry personnel and engineering students. Global J Eng Educ. 1998;2(1):65-79.

[9] Lord SM The borderlands of education: Latinas in engineering. Lanham (MD): Lexington Books; 2013.

[10] Vinlove S. Learning to teach where you are: preparation for context-responsive teaching in Alaska's teacher certification programs [PhD dissertation]. Fairbanks: University of Alaska; 2002. Available from http://ankn.uaf.edu/Curriculum/PhD_Projects/AmyVinlove/Vinlove.pdf

[11] Hiebert J, Lefevre P. Procedural and conceptual knowledge in mathematics: an introductory analysis. In: J Hiebert, editor. Conceptual and procedural knowledge: the case of mathematics. Hillsdale (NJ): Erlbaum; 1986. p. 1-27.

[12] Hiebert J, Carpenter T. Learning and teaching with understanding. In: D Grouws, editor. Handbook of research on mathematics teaching and learning. New York: Macmillan Publishing Company. 1992. p. 65-97.

[13] Skemp R. Relational and instrumental understanding. Arithmetic Teach. 1978;26:9-15.

[14] Peled I, Zaslavski O. Beyond local conceptual connections: meta-knowledge about procedures. Learn Math. 2008;28(3):28-35.

[15] Rittle-Johnson B, Alibali MW. Conceptual and procedural knowledge of mathematics: does one lead to another? J Educ Psychol. 1999;91(1):175-189.

[16] Artigue M. Digital technologies: a window on theoretical issues in mathematics education. In: Pitta-Pantazi D, Philippou G, editors. European research in mathematics education V. Proceedings of CERME6. Larnaca: University of Cyprus. 2007. p. 68-82.

[17] Baker W, Czarnocha B. Written meta-cognition and procedural knowledge. In: Vakalis I, et al., editors. Proceedings of the 2nd International Conference on the Teaching of Mathematics (at the undergraduate level); July 2002. Hersonissos (Greece): University of Crete; 2002. 
[18] Radu M. Basic skills versus conceptual understanding in mathematics education: the case of fractions and division. A reply to Hung-Hsi Wu. ZDM. 2002;34(3):93-95.

[19] Silver E. Using conceptual and procedural knowledge: a focus on relationships. In: Hiebert J, editor. Conceptual and procedural knowledge: the case of mathematics. Hillsdale (NJ): Erlbaum; 1986. p. 181-198.

[20] Bergsten C. Matematisk operativitet. En analys av relationen mellan form och innehåll i skolmatematik. [Mathematical operativity. An analysis of the form-content relationship in school mathematics] [Doctoral thesis]. Linköping: Linköping University; 1990.

[21] Sfard A. On the dual nature of mathematical conceptions: reflections on processes and objects as different sides of the same coin. Educ Stud Math. 1991;22(1):1-36.

[22] Star JR. Reconceptualizing procedural knowledge. J Res Math Educ. 2005;36(5):404-411.

[23] Baroody AJ, Feil Y, Johnson AR. An alternative reconceptualization of procedural and conceptual knowledge. J Res Math Educ. 2007;38(2):115-131.

[24] Gray E, \& Tall D Duality, ambiguity and flexibility: a proceptual view of simple arithmetic. J Res Math Educ. 1994;26(2):115-141.

[25] Kieran C. The false dichotomy in mathematics education between conceptual understanding and procedural skills: an example from algebra. In: Leatham KR, editor. Vital directions for mathematics education research. New York (NY): Springer; 2013. p. 153-171.

[26] Wu H. Basic skills versus conceptual understanding: a bogus dichotomy in mathematics education. Am Educ. 1999. Fall 1999:1-7. Available from: www.aft.org/pdfs/americaneducator/ fall1999/wu.pdf.

[27] Engelbrecht J, Bergsten C, Kågesten O. Undergraduate students' preference for procedural to conceptual solutions to mathematical problems. Int J Math Educ Sci Technol. 2009;40(7):927940.

[28] Engelbrecht J, Bergsten C, Kågesten O. Conceptual and procedural approaches to mathematics in the engineering curriculum: student conceptions and performance. J Eng. Educ. 2012;101(1):138-162.

[29] Bergsten C, Engelbrecht J, Kågesten O. Conceptual and procedural approaches to mathematics in the engineering curriculum: a comparison between conceptions and performance of junior and senior engineering students in two countries. (Submitted).

[30] Engelbrecht J, Harding A, Potgieter M. Undergraduate students' performance and confidence in procedural and conceptual mathematics. Int J Math Educ Sci Technol. 2005;36(7):701-712.

[31] Engelbrecht J, Bergsten C, Kågesten O. Conceptual and procedural approaches to mathematics in the engineering curriculum: views of qualified engineers. (Submitted).

[32] Bryman A Social research methods. 2nd ed. Oxford: Oxford University Press; 2004.

[33] Geertz C Thick description: toward an interpretive theory of culture. In: C Geertz, editor. The interpretation of cultures: selected essays. New York (NY): Basic Books; 1973. p. 3-30. 